\title{
Development and external validation of a nomogram for predicting the effect of tumor size on survival of patients with perihilar cholangiocarcinoma
}

Yaodong Zhang ${ }^{1 \dagger}$, Zhengshan $\mathrm{Wu}^{1+}$, Xing Wang ${ }^{1,2+}$, Changxian $\mathrm{Li}^{1}$, Jiang Chang ${ }^{1}$, Wangjie Jiang ${ }^{1}$, Hongwei Wang ${ }^{1}$, Yirui Wang ${ }^{1}$ and Xiangcheng $\mathrm{Li}^{i^{*}}$

\begin{abstract}
Background: The effect of tumor size on account of long-term survival results in perihilar cholangiocarcinoma (PCCA) patients has remained a controversial debate. It is urgent necessary to identify the optimal cutoff value of tumor size in PCCA and integrate tumor size with other prognostic factors into a nomogram to improve the predictive accuracy of prognosis of patients with PCCA.

Methods: Three hundred sixty-three PCCA patients underwent surgical resection were extracted from the Surveillance, Epidemiology and End Results (SEER) database. X-tile program was used to identify the optimal cutoff value of tumor size. A nomogram including tumor size was established to predict 1-, 3- and 5-year cancer-specific survival (CSS) based on the independent risk factors chosen by Kaplan-Meier methods and multivariable cox regression models. The precision of the nomogram for predicting survival was validated internally and externally.

Results: PCCA patients underwent surgical resection were classified into $1-19 \mathrm{~mm}, 20-33 \mathrm{~mm}$ and $\geq 34 \mathrm{~mm}$ subgroups based on the optimal cutoff for tumor size in terms of CSS. And we noticed that more larger tumor size group had worse tumor grade, advanced T stage, more positive regional lymph nodes and more frequent vascular invasion. The nomogram according to the independent factors was well calibrated and displayed better discrimination power than 7th Tumor-Node-Metastasis (TNM) stage systems.
\end{abstract}

Conclusions: The results demonstrated that the larger tumor size of PCCA was, the worse survival would be. The proposed nomogram, which outperforms the conventional TNM staging system, showed relatively good performance and could be considered as convenient individualized predictive tool for prognosis of PCCA patients.

Keywords: Nomogram, Tumor size, Perihilar cholangiocarcinoma

\footnotetext{
* Correspondence: drxcli@njmu.edu.cn

†Yaodong Zhang, Zhengshan Wu and Xing Wang these authors contributed

to the work equally and should be regarded as co-first authors

'Key Laboratory on Living Donor Transplantation, Ministry of Health,

Department of liver surgery, The First Affiliated Hospital of Nanjing Medical University, 300\# Guangzhou Road, Nanjing 210029, Jiangsu Province, China

Full list of author information is available at the end of the article
}

(c) The Author(s). 2020 Open Access This article is licensed under a Creative Commons Attribution 4.0 International License, which permits use, sharing, adaptation, distribution and reproduction in any medium or format, as long as you give appropriate credit to the original author(s) and the source, provide a link to the Creative Commons licence, and indicate if changes were made. The images or other third party material in this article are included in the article's Creative Commons licence, unless indicated otherwise in a credit line to the material. If material is not included in the article's Creative Commons licence and your intended use is not permitted by statutory regulation or exceeds the permitted use, you will need to obtain permission directly from the copyright holder. To view a copy of this licence, visit http://creativecommons.org/licenses/by/4.0/ The Creative Commons Public Domain Dedication waiver (http://creativecommons.org/publicdomain/zero/1.0/) applies to the data made available in this article, unless otherwise stated in a credit line to the data. 


\section{Background}

Perihilar cholangiocarcinoma (PCCA) remains one of the most dismal malignancy involving the confluence of the hepatic ducts [1]. Curative-intent resection is still the only effective curative option for PCCA patients that are detected at early stages. However, due to the conceal clinical symptom, most patients are admitted with macrovascular invasion, lymph nodes or liver parenchyma involvement, resulting only $10-15 \%$ patients can be amendable to resection with curative intent [2]. Even for the patients underwent surgical resection, the 5-year survival rate remains a disappointing 20-35\% [3-8].

Unlike lymph node metastasis and vascular invasion, which have been recognized as the independent prognostic factors in PCCA, the impact of tumor size on account of the long-term survival results in PCCA patients, especially for those who underwent resection, has remained a worldwide controversial debate. Several studies have found no association of tumor size with survival [9-13], while others have reported that tumor size smaller than $3 \mathrm{~cm}$ showed better outcome [14-18]. This divergence is also existed in different staging systems. Tumor-Node-Metastasis (TNM) staging system of the American Joint Commission on Cancer (AJCC), which is the wide-accepted standard for PCCA, does not take tumor size into account, whereas the DeOliveira staging system labels tumor size as T1 $(1 \mathrm{~cm})$, T2 (1$3 \mathrm{~cm})$ and $\mathrm{T} 3(\geq 3 \mathrm{~cm})$ for predicting PCCA patients prognosis [14]. Therefore, we sought to explore the prognostic value of tumor size in PCCA based on data from Surveillance, Epidemiology, and End Results (SEER) Program of the National Cancer Institute.

Due to prognosis prediction is a pivotal reference factor in clinical personalized treatment, a nomogram is an optimal tool, which can incorporate risk factors involved in tumor development, for visually estimating the survival probability of patients based on a statistical predictive model. To our best knowledge, there is no published study reported the nomogram for predicting the prognosis of PCCA based on tumor size. The aims of our study were to evaluate the optimal cutoff value of tumor size in PCCA, and conduct a nomogram incorporated important factors obtained from SEER database for predicting the probability of cancer-specific survival (CSS) of resectable PCCA patients, and externally validating the prognostic model based on the data from the First Affiliated Hospital of Nanjing Medical University.

\section{Methods}

\section{Data source and selection}

Data of patients diagnosed with PCCA were obtained from SEER 18 registries research database (1975-2016), which provides cancer incidence and survival information among approximately $30 \%$ of the population in the
United States. To ensure sufficient time for follow-up and integrity of clinical information, we selected patients included in TNM 7/CS v0204+ Schema BileDuctsPerihilar between 2004 and 2015. On the basis of excluding carcinoid tumor, neuroendocrine carcinoma, and adenosarcoma, we also identified PCCA using ICD-O-3 histology codes reported in Mao et al.: 8000-8152, 81548231, 8243-8245, 8250-8576, 8940-8950, and 89808981 [19]. And CS Site-Specific Factor 25 (Schema Discriminator: BileDuctsDistal/ BileDuctsPerihilar/CysticDuct) codes 010,020,050,060,100 were applied to further define the location of the tumor in the hilum. Patients were also excluded if they were only diagnosed as PCCA clinically, had incomplete survival time or PCCA was not primary cancer. To explore the prognostic value of tumor size in resectable PCCA, we excluded patients with unknown tumor size, no evidence of primary lesion and not perform the surgical resection. Based on ensuring enough samples for analysis, we excluded patients with incomplete information in such aspects as surgery, regional lymph node detection and race, so as to improve the practical value of nomogram in clinical. The stepwise extraction process of available cases as shown in Fig. 1, a total of 363 cases matching the inclusion criteria were finally chosen in this analysis.

To estimate the generalizability of the novel nomogram based on SEER database. We performed the separate external validation using 132 eligible PCCA cases diagnosed in the First Affiliated Hospital of Nanjing Medical University. The clinicopathological features of all patients are shown in Table 1. The investigation was approved by the ethics committee of the First Affiliated Hospital of Nanjing Medical University.

\section{Identification of the optimal cutoff value of tumor size in PCCA}

To investigate association of tumor size with survival, Xtile program, which was reported previously [20], was used to divide PCCA patients into three group according to tumor size by log-rank $\chi^{2}$ statistics in terms of CSS. And the optimal cutoff values were validated via internal and external validation.

\section{Statistical analysis}

Information about study cohort collected from SEER database were used to identify the cutoff for the categorical tumor size in terms of survival and develop prognostic nomogram. Nine demographic information and clinicopathological features, including age at diagnosis, race, sex, tumor grade, $\mathrm{T}$ stage, $\mathrm{M}$ stage (Derived AJCC Stage Group, 7th ed. (2010-2015)), regional lymph nodes status (combined Collaborative Stage Data Collection System (CS) regional nodes examined and CS regional nodes positive), vascular invasion (CS extension) 


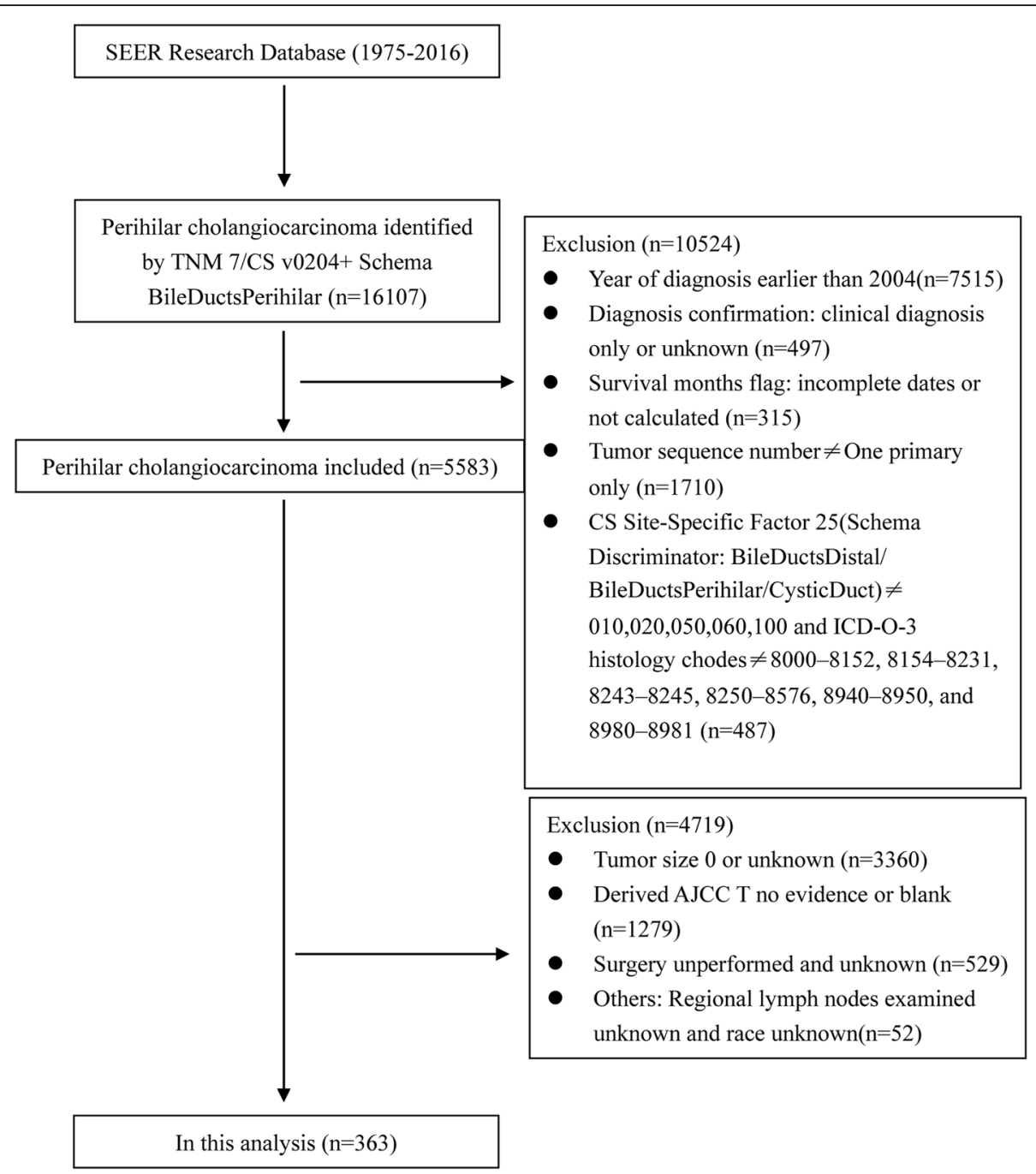

Fig. 1 Flowchart displaying the extraction process of resected PCCA cases in SEER database

and tumor size (CS tumor size, 2004+) were used to conduct the analysis.

We evaluated the prognostic effect of each clinical variable using cumulative survival curves and log-rank test calculate by Kaplan-Meier method. CSS was set as the main prognostic indicator of patients with PCCA. CSS was defined as the length of time from data of diagnosis to death due to PCCA. Cox proportional hazard models were applied to filter out significant variables, and the independent risk factors chosen by multivariate analysis were incorporated to construct the nomogram for predicting 1-,3- and 5- year CSS. The construct nomogram was subjected internal validation through 1000 bootstrap resamples and further underwent external validated with the 132 patients from the First Affiliated Hospital of Nanjing Medical University. The area under receiver operating characteristic (ROC) curve (AUC) and the consistency index (C-index) were used to verify the precision of nomogram. Meanwhile, calibration plotting was used to evaluate the agreement between the actual outcome and the predicted probability. Decision Curve Analysis (DCA), as a suitable method for evaluating alternative diagnostic and prognostic strategies, was also used to assess the accuracy of nomogram compared to the conventional TNM staging system. All statistical analyses were conducted on SPSS version 21.0 (SPSS Inc., Chicago, IL, USA) and R software 3.6.2 (R foundation, Vienna, Austria). A two tailed $p$-value $<0.05$ was considered statistically significant.

\section{Results}

Patients characteristics and survival outcomes

In total, this study involved 363 eligible patients diagnosed with PCCA as the sole primary cancer and underwent surgical resection in SEER database from 2004 to 2015. The descriptive and clinical characteristics of these 
Table 1 Patients characteristics

\begin{tabular}{|c|c|c|c|c|c|c|}
\hline \multirow[t]{2}{*}{ Characteristic } & & \multicolumn{2}{|c|}{ Training cohort } & \multicolumn{2}{|c|}{ Validation cohort } & \multirow[t]{2}{*}{$P$ value } \\
\hline & & No.patients & $\%$ & No.patients & $\%$ & \\
\hline \multirow[t]{2}{*}{ Age } & $<60$ & 106 & 29.2 & 44 & 33.3 & 0.376 \\
\hline & $\geq 60$ & 257 & 70.8 & 88 & 66.7 & \\
\hline \multirow[t]{3}{*}{ Race } & White & 265 & 73.0 & & & \\
\hline & Black & 26 & 7.2 & & & \\
\hline & Others & 72 & 19.8 & & & \\
\hline \multirow[t]{2}{*}{ Sex } & Female & 136 & 37.5 & 41 & 31.1 & 0.204 \\
\hline & Male & 227 & 62.5 & 91 & 68.9 & \\
\hline \multirow[t]{3}{*}{ Grade } & Well/moderate & 217 & 59.8 & 61 & 46.2 & $<0.001$ \\
\hline & Poor/undifferentiated & 102 & 28.1 & 68 & 51.5 & \\
\hline & Unknown & 44 & 12.1 & 3 & 2.3 & \\
\hline \multirow[t]{5}{*}{ T stage } & $\mathrm{T} 1$ & 55 & 15.2 & 8 & 6.1 & $<0.001$ \\
\hline & $\mathrm{T} 2$ & 175 & 48.2 & 80 & 60.6 & \\
\hline & T3 & 50 & 13.8 & 30 & 22.7 & \\
\hline & T4 & 24 & 6.6 & 10 & 7.6 & \\
\hline & $T x$ & 59 & 16.3 & 4 & 3.0 & \\
\hline \multirow[t]{2}{*}{ M stage } & Mo & 345 & 95 & 130 & 98.5 & 0.119 \\
\hline & M1 & 18 & 5 & 2 & 1.5 & \\
\hline \multirow[t]{4}{*}{ RN status } & RN examined and not positive & 169 & 46.6 & 71 & 53.8 & 0.253 \\
\hline & RN examined and $<4$ nodes positive & 114 & 31.4 & 40 & 30.3 & \\
\hline & RN examined and $>=4$ nodes positive & 34 & 9.4 & 12 & 9.1 & \\
\hline & RN not examined or RN unknown & 46 & 12.7 & 9 & 6.8 & \\
\hline \multirow[t]{2}{*}{ Vascular Invasion } & Absent & 315 & 86.8 & 84 & 63.6 & 0.399 \\
\hline & Present & 48 & 13.2 & 48 & 36.4 & \\
\hline \multirow[t]{3}{*}{ Tumor size } & $1-19 \mathrm{~mm}$ & 88 & 24. & 29 & 22.0 & 0.871 \\
\hline & $20-33 \mathrm{~mm}$ & 160 & 44.1 & 60 & 45.5 & \\
\hline & $\geq 34 \mathrm{~mm}$ & 115 & 31.7 & 43 & 32.6 & \\
\hline
\end{tabular}

RN Regional lymph nodes

patients were provided in Table 1 . In the training cohort, the median follow-up periods were 23.87 (range, 1-83) months with $70.8 \%$ patients being over age of 60 years. Notably, in this study, we combined the variables ("regional nodes examined" and "regional nodes positive", which were included in Collaborative Stage Data Collection System) into regional lymph nodes status, which was divided into four subgroups: regional nodes examined and not positive, regional nodes examined and $<4$ nodes positive, regional nodes examined and $\geq 4$ nodes positive, and regional nodes not examined or regional nodes unknown. 78\% resected PCCA patients had less than 4 positive regional lymph nodes. We also noticed that $86.6 \%$ patients had no vascular invasion, which was seem as the important factor in prognosis of PCCA. In general, in terms of prognosis, the 1-,3- and 5- year CSS rates were stratified by age, sex, tumor grade, $\mathrm{T}$ stage, and $M$ stage (7th edition), regional lymph nodes status, vascular invasion and tumor size.

\section{Identification of optimal tumor-size cutoff value with prognosis}

The cutoff values, $20 \mathrm{~mm}$ and $34 \mathrm{~mm}$, of tumor size were identified by X-tile plot based on minimal $P$-value approach and the maximum of chi-square log-rank value was 24.1 according to cancer-specific survival (Fig. 2a). To investigate the impact of tumor-size cutoff values on CSS, we first reclassified patients into three risk groups, $1-19 \mathrm{~mm}, 20-33 \mathrm{~mm}$ and $\geq 34 \mathrm{~mm}$, using $20 \mathrm{~mm}$ and $34 \mathrm{~mm}$ as the cutoff values. The tumor size is an important prognosis factor for PCCA, and patients with $34 \mathrm{~mm}$ or more had significant poor prognosis, and $20 \mathrm{~mm}$ or less group showed better survival outcome than other groups in CSS $(p<0.001$, Fig. $2 \mathrm{~b})$. In the external validation cohort, we also found that tumor size less than 20 $\mathrm{mm}$ in PCCA patients had a better survival outcome, and tumor size larger than $34 \mathrm{~mm}$ is a significant negative factor implying a worse prognosis (Fig. 2c). Then, to 


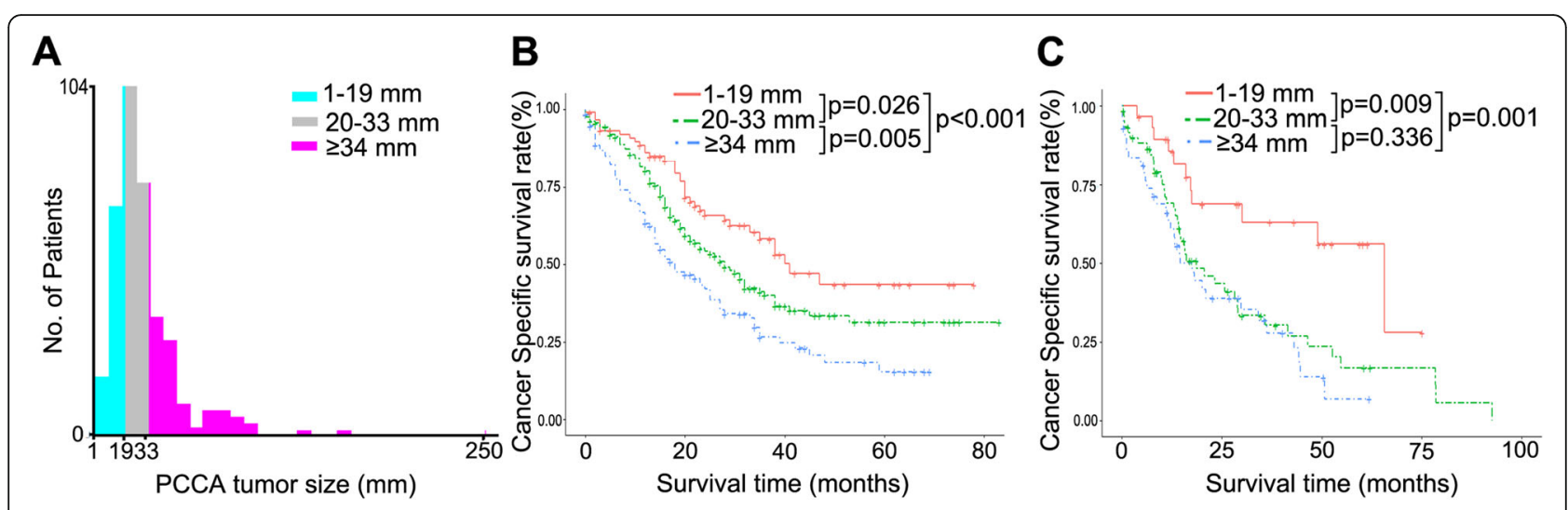

Fig. 2 X-tile analysis was done on patient data from the SEER registry. a is shown on a histogram of the entire cohort. Cancer-specific survival rates for PCCA patients in training cohort (b) and external validation cohort (c)

further confirm the impact of different tumor size on CSS of resected PCCA, we treated the size of PCCA as a continuous variable and analyzed the size of PCCA from 18 to $40 \mathrm{~mm}$ (Table 2). The tumor size was the independent factor for PCCA, and patients with tumor size more than $34 \mathrm{~mm}$ had significant worse 5-year survival outcome and shorter mean survival time than other groups. Notably, when the tumor size was $33 \mathrm{~mm}$ and less, the difference in the prognosis of patients was reduced compared with others. Until the cut-off value was $20 \mathrm{~mm}$, the prognosis of patients with tumor size greater than $20 \mathrm{~mm}$ was significantly worse than that with tumor size less than $20 \mathrm{~mm}$. To better reveal the clinical value of these tumor-size cutoff values, we compared the differences in tumor characteristics among the three subgroups. Of these, we noticed that more larger tumor size group had worse tumor grade, advanced $\mathrm{T}$ stage, more positive regional lymph nodes and more frequent vascular invasion (Table 3 , all $p<0.05$ ).

Effect of tumor size counts on survival outcome of PCCA Initially, univariate analysis revealed that, besides of tumor size, other clinicopathologic characteristics strongly correlated with CSS with tumor grade, T stage and regional lymph nodes status (Table 4). Multivariate analysis with cox regression demonstrated that more larger tumor size showed survival disadvantage in CSS (tumor size: $20-33 \mathrm{~mm}$ hazard ratio (HR) 1.525 ; $\geq 34 \mathrm{~mm}$ HR 2.354). Notably, we noticed that tumor size less than $20 \mathrm{~mm}$ in PCCA patients indicated a better prognosis, while patients with tumor size larger than $34 \mathrm{~mm}$ were a significant negative factor affecting prognosis. Of other variables, tumor grade, $\mathrm{T}$ stage and regional lymph nodes status remained significantly associated with CSS in the multivariate cox regression model (all $P<0.05$ ), suggesting they were also the independent predictors for CSS in resected PCCA patients (Table 4).

\section{Construction and validation of Nomogram for CSS}

With the independent predictors of CSS derived from multivariate analysis, we established a nomogram to predict the 1-,3- and 5-year CSS in patients with PCCA. Tumor grade, regional lymph nodes status and tumor size were included into the nomogram (Fig. 3a). Notably, the tumor size contributed the most to prognosis followed by regional lymph nodes status and tumor grade in the novel nomogram. Each variable in the nomogram was assigned a risk score on the point scale. And we were able to calculate the total risk points to estimate 1-, 3- and 5-year survival rates according to the survival probability scale in the nomogram. The nomogram based on tumor size showed good accuracy with c-index of 0.626 for CSS in the training cohort. Calibration plots for the probabilities of 1-, 3-, and 5-year CSS showed the optimal agreement between predictions by the nomogram and the actual observations regarding both training and external validation sets (Fig. 3). To further explore the discriminatory accuracy of the nomogram with that of 7th edition TNM staging systems in the training set. The discriminatory accuracy of the nomogram for CSS prediction was superior to that of 7th edition TNM stage systems (C-index: 0.626 vs 0.608$)$. The external prognostic validation using the above cut-points revealed that prognosis of patients with tumor size less than $20 \mathrm{~mm}$ had more better survival outcome, and the calibration plots also performed the good correlations between the nomogram predictions and actual observations for 1-, 3- and 5-year CSS, and C-index of the nomogram was also higher than the 7th edition TNM stage systems (C-index: 0.606 vs 0.541 , Fig. 3).

\section{Comparison of the values of area under the ROC curve}

Comparison of the novel nomogram and the 7th edition TNM staging system was performed using the AUC values. For the whole study cohort, the AUC values of the nomogram for predicting 1-,3- and 5- year CSS were $0.680,0.691$ and 0.727 . These results were significantly 
Table 2 Univariate analysis for the influence of different cutoffs of tumor size on CSS in perihilar cholangiocarcinoma

\begin{tabular}{|c|c|c|c|c|c|c|c|c|}
\hline Cutoff & No. & 5-year CSS (\%) & Difference of CSS (\%) & Log-rank $x^{2}$ & $P$ value & Median of CSS (Months) & Difference of Median (Months) & HR \\
\hline$<18$ & 71 & 47.9 & 23.2 & 7.407 & 0.006 & 41 & 16 & 1.705 \\
\hline$\geq 18$ & 292 & 24.7 & & & & 25 & & \\
\hline$<19$ & 85 & 42.6 & 17.7 & 9.443 & 0.002 & 40 & 16 & 1.737 \\
\hline$\geq 19$ & 278 & 24.9 & & & & 24 & & \\
\hline$<20$ & 88 & 43.5 & 19.1 & 11.37 & 0.001 & 41 & 17 & 1.83 \\
\hline$\geq 20$ & 275 & 24.4 & & & & 24 & & \\
\hline$<21$ & 122 & 37.9 & 13.4 & 9.716 & 0.002 & 38 & 15 & 1.617 \\
\hline$\geq 21$ & 241 & 24.5 & & & & 23 & & \\
\hline$<22$ & 130 & 37.3 & 13.1 & 9.474 & 0.002 & 38 & 15 & 1.589 \\
\hline$\geq 22$ & 233 & 24.2 & & & & 23 & & \\
\hline$<23$ & 143 & 36.4 & 12.5 & 10.119 & 0.001 & 35 & 12 & 1.59 \\
\hline$\geq 23$ & 220 & 23.9 & & & & 23 & & \\
\hline$<24$ & 152 & 36.9 & 13.8 & 11.094 & 0.001 & 35 & 13 & 1.614 \\
\hline$\geq 24$ & 211 & 23.1 & & & & 22 & & \\
\hline$<25$ & 162 & 36.2 & 13.1 & 9.885 & 0.002 & 35 & 12 & 1.561 \\
\hline$\geq 25$ & 201 & 23.1 & & & & 23 & & \\
\hline$<26$ & 187 & 33.9 & 10 & 7.067 & 0.008 & 33 & 10 & 1.477 \\
\hline$\geq 26$ & 176 & 23.9 & & & & 23 & & \\
\hline$<27$ & 189 & 35.2 & 12.8 & 9.061 & 0.003 & 35 & 12 & 1.518 \\
\hline$\geq 27$ & 174 & 22.4 & & & & 23 & & \\
\hline$<28$ & 195 & 34.9 & 12.5 & 9.97 & 0.002 & 35 & 13 & 1.548 \\
\hline$\geq 28$ & 168 & 22.4 & & & & 22 & & \\
\hline$<29$ & 200 & 35.3 & 13.4 & 9.969 & 0.002 & 33 & 11 & 1.547 \\
\hline$\geq 29$ & 163 & 21.9 & & & & 22 & & \\
\hline$<30$ & 202 & 34.9 & 12.7 & 8.494 & 0.004 & 33 & 11 & 1.497 \\
\hline$\geq 30$ & 161 & 22.2 & & & & 22 & & \\
\hline$<31$ & 236 & 33.6 & 12.9 & 8.859 & 0.003 & 32 & 10 & 1.52 \\
\hline$\geq 31$ & 127 & 20.7 & & & & 22 & & \\
\hline$<32$ & 240 & 33.9 & 14.3 & 9.037 & 0.003 & 32 & 10 & 1.529 \\
\hline$\geq 32$ & 123 & 19.6 & & & & 22 & & \\
\hline$<33$ & 246 & 35.1 & 18.5 & 13.726 & 0 & 32 & 14 & 1.687 \\
\hline$\geq 33$ & 117 & 16.6 & & & & 18 & & \\
\hline$<34$ & 248 & 35.8 & 20.4 & 16.741 & 0 & 32 & 14 & 1.78 \\
\hline$\geq 34$ & 115 & 15.4 & & & & 18 & & \\
\hline$<35$ & 248 & 35.8 & 20.4 & 16.741 & 0 & 32 & 14 & 1.78 \\
\hline$\geq 35$ & 115 & 15.4 & & & & 18 & & \\
\hline$<36$ & 264 & 34.2 & 19 & 13.681 & 0 & 32 & 14 & 1.715 \\
\hline$\geq 36$ & 99 & 15.2 & & & & 18 & & \\
\hline$<37$ & 213 & 35.1 & 13.6 & 7.73 & 0.005 & 32 & 9 & 1.469 \\
\hline$\geq 37$ & 150 & 21.5 & & & & 22.5 & & \\
\hline$<38$ & 214 & 34.9 & 13.2 & 7.263 & 0.007 & 24.8 & 2.3 & 1.452 \\
\hline$\geq 38$ & 149 & 21.7 & & & & 22.5 & & \\
\hline$<39$ & 217 & 34.4 & 12.3 & 5.362 & 0.021 & 24.6 & 1.7 & 1.379 \\
\hline$\geq 39$ & 146 & 22.1 & & & & 22.9 & & \\
\hline$<40$ & 277 & 33 & 16.4 & 7.667 & 0.006 & 24.9 & 4.4 & 1.527 \\
\hline$\geq 40$ & 86 & 16.6 & & & & 20.5 & & \\
\hline
\end{tabular}


Table 3 Baseline demographic and tumor characteristics of patients based on tumor size

\begin{tabular}{|c|c|c|c|c|c|c|}
\hline \multirow[t]{2}{*}{ Characteristic } & & \multicolumn{3}{|c|}{ Tumor size } & \multirow[b]{2}{*}{ Log-rank } & \multirow[b]{2}{*}{$P$ value } \\
\hline & & $1-19 \mathrm{~mm}$ & $20-33 \mathrm{~mm}$ & $\geq 34 \mathrm{~mm}$ & & \\
\hline \multirow[t]{2}{*}{ Age } & $<60$ & $26(29.5)$ & $48(30.0)$ & $32(27.8)$ & 0.16 & 0.923 \\
\hline & $\geq 60$ & $62(70.5)$ & $112(70.0)$ & $83(72.2)$ & & \\
\hline \multirow[t]{3}{*}{ Race } & White & $63(71.6)$ & $123(76.9)$ & $79(68.7)$ & 0.401 & 4.035 \\
\hline & Black & $5(5.7)$ & $9(5.6)$ & $12(10.4)$ & & \\
\hline & Others & $20(22.7)$ & $28(17.5)$ & $24(20.9)$ & & \\
\hline \multirow[t]{2}{*}{ Sex } & Female & $29(33.0)$ & $56(35.0)$ & $51(44.3)$ & 3.504 & 0.173 \\
\hline & Male & $59(67.0)$ & $104(65.0)$ & $64(55.7)$ & & \\
\hline \multirow[t]{3}{*}{ Grade } & Well/moderate & $53(60.2)$ & $102(63.8)$ & $62(53.9)$ & 10.169 & 0.038 \\
\hline & Poor/undifferentiated & $18(20.5)$ & $45(28)$. & $39(33.9)$ & & \\
\hline & Unknown & $17(19.3)$ & $13(8.1)$ & $14(12.2)$ & & \\
\hline \multirow[t]{5}{*}{ T5 } & $\mathrm{T} 1$ & $24(27.3)$ & $20(12.5)$ & $11(9.6)$ & 28.336 & $<0.001$ \\
\hline & $\mathrm{T} 2$ & $37(42.0)$ & $80(50.0)$ & $58(50.4)$ & & \\
\hline & $\mathrm{T} 3$ & $10(11.4)$ & $21(13.1)$ & 19 (16.5) & & \\
\hline & $\mathrm{T} 4$ & $2(2.3)$ & $7(4.4)$ & 15 (13.0) & & \\
\hline & Tx & $15(17.0)$ & $32(20.0)$ & $12(10.4)$ & & \\
\hline \multirow[t]{2}{*}{ M } & Mo & 85 (96.6) & $153(95.6)$ & $107(93.0)$ & 1.538 & 0.463 \\
\hline & M1 & $3(3.4)$ & $7(4.4)$ & $8(7.0)$ & & \\
\hline \multirow[t]{4}{*}{ RN status } & RN examined and not positive & $52(59.1)$ & $76(47.5)$ & $41(35.7)$ & 15.744 & 0.015 \\
\hline & RN examined and $<4$ nodes positive & $21(23.9)$ & $53(33.1)$ & $40(34.8)$ & & \\
\hline & RN examined and $>=4$ nodes positive & $3(3.4)$ & $15(9.4)$ & $16(13.9)$ & & \\
\hline & RN not examined or RN unknown & $12(13.6)$ & $16(10.0)$ & $18(15.7)$ & & \\
\hline \multirow[t]{2}{*}{ Vascular Invasion } & Absent & $83(94.3)$ & $141(88.1)$ & $91(79.1)$ & 10.475 & 0.005 \\
\hline & Present & $5(5.7)$ & $19(11.9)$ & $24(20.9)$ & & \\
\hline
\end{tabular}

better than the corresponding 1- and 5-year AUC values of CSS in TNM staging system (AUC value: 1-year 0.609, 3-year 0.671 and 5-year 0.628, Fig. 4a, b \& c). In the external cohort, the1-, 3- and 5-year AUC values of the nomogram also showed better accuracy for predicting the CSS than that with the 7th TNM stage system ( 0.632 vs $0.572,0.655$ vs 0.527 and 0.709 vs 0.540 , Fig. $4 d$, e \& f). DCA was performed to compare the clinical usability and benefits of the nomogram with that of the traditional AJCC stage. As shown in Fig. 4g \& h, compared to the AJCC stage model, the new nomogram's 5year DCA curves showed larger net benefits across a range of death risk in the training and external validation cohort.

\section{Discussion}

Current curative therapeutic option is limited to surgical treatment which has disappointing outcome with a 5year survival rate less than 30\% [21]. Among the prognostic factors, such as lymph nodal status and resection margin status, influencing the prognosis of PCCA, the impact of tumor size on survival and its clinical value have been still controversial so far. Tumor size was not included in TNM staging system, which is mainly based on the pathological findings. However, different researchers have different views about the effect of tumor size in predicting outcome of PCCA. DeOliveira staging system [22] divided the tumor size into 3 subgroups, T1 $(1 \mathrm{~cm}), \mathrm{T} 2(1-3 \mathrm{~cm})$ and $\mathrm{T} 3(\geq 3 \mathrm{~cm})$, and emphasized 3 $\mathrm{cm}$ cutoff for T3 has worse prognosis compared to smaller tumors $[11,12,14]$. In other studies that supported tumor size should be a prognostic indicator, some researchers suggested 2-cm cutoff is the optimal tumor-size cutoff point.

The nomogram is a visualization tool for calculating the probability of survival for various cancers and has been widely accepted in clinical practice with its feasibility and accuracy [23-25]. Thus, we attempted to establish a prognostic nomogram to predict CSS at 1-, 3- and 5- years in PCCA patients. Via the construction of nomogram, we first used the $\mathrm{x}$-tile program to identify $20 \mathrm{~mm}$ and $34 \mathrm{~mm}$ as the optimal cutoff value for tumor 
Table 4 Univariate and multivariate cancer-specific survival analyses for the influence of tumor size in perihilar cholangiocarcinoma

\begin{tabular}{|c|c|c|c|c|c|}
\hline \multirow[t]{2}{*}{ Variable } & & \multicolumn{2}{|l|}{ Univariable analysis } & \multicolumn{2}{|c|}{ Multivariable analysis } \\
\hline & & HR (95\% CI) & $P$ & HR (95\% Cl) & $P$ \\
\hline \multirow[t]{2}{*}{ Age } & $<60$ & Reference & 0.060 & & \\
\hline & $\geq 60$ & $1.354(0.988-1.858)$ & & & \\
\hline \multirow[t]{3}{*}{ Race } & White & Reference & 0.125 & & \\
\hline & Black & $1.165(0.684-1.984)$ & & & \\
\hline & Others & $1.419(1.011-1.991)$ & & & \\
\hline \multirow[t]{2}{*}{ Sex } & Female & Reference & & & \\
\hline & Male & $0.939(0.708-1.247)$ & 0.666 & & \\
\hline \multirow[t]{3}{*}{ Grade } & Well/moderate & Reference & $<0.001$ & Reference & 0.003 \\
\hline & Poor/undifferentiated & $1.899(1.401-2.574)$ & & $1.733(1.262-2.379)$ & \\
\hline & Unknown & $1.255(0.31-1.897)$ & & $1.335(0.860-2.073)$ & \\
\hline \multirow[t]{5}{*}{ T stage } & $\mathrm{T} 1$ & Reference & 0.007 & Reference & 0.170 \\
\hline & $\mathrm{T} 2$ & $1.634(1.043-2.558)$ & & $1.209(0.756-1.932)$ & \\
\hline & T3 & $1.997(1.164-3.426)$ & & $1.063(0.599-1.886)$ & \\
\hline & T4 & $2.655(1.424-4.951)$ & & $1.411(0.735-2.711)$ & \\
\hline & Tx & $2.324(1.3753 .929)$ & & $1.801(1.032-3.143)$ & \\
\hline \multirow[t]{2}{*}{ M stage } & Mo & Reference & 0.063 & & \\
\hline & M1 & $1.676(0.973-2.887)$ & & & \\
\hline \multirow[t]{4}{*}{ RN status } & RN examined and not positive & Reference & $<0.001$ & Reference & $<0.001$ \\
\hline & RN examined and $<4$ nodes positive & $2.294(1.658-3.175)$ & & $2.168(1.551-3.031)$ & \\
\hline & RN examined and $>=4$ nodes positive & $2.968(1.857-4.743)$ & & $2.592(1.562-4.302)$ & \\
\hline & RN not examined or RN unknown & $2.026(1.317-3.119)$ & & $1.952(1.254-3.039)$ & \\
\hline \multirow[t]{2}{*}{ Vascular Invasion } & Absent & Reference & 0.062 & & \\
\hline & Present & $1.432(0.981-2.090)$ & & & \\
\hline \multirow[t]{3}{*}{ Tumor size } & $1-19 \mathrm{~mm}$ & Reference & $<0.001$ & Reference & 0.008 \\
\hline & $20-33 \mathrm{~mm}$ & $1.525(1.034-2.249)$ & & $1.264(0.844-1.894)$ & \\
\hline & $\geq 34 \mathrm{~mm}$ & $2.354(1.586-3.494)$ & & $1.834(1.209-2.780)$ & \\
\hline
\end{tabular}

RN Regional lymph nodes

size in terms of CSS. The results were then further internal and external validated by Kaplan-Meier method to reveal that tumor size within $20 \mathrm{~mm}$ showed better prognosis in PCCA patients, and patients with tumors size beyond $34 \mathrm{~mm}$ had disappointing survival outcome. Notably, our results demonstrated that more larger tumor size group had worse tumor grade, advanced $\mathrm{T}$ stage, more positive regional lymph nodes and more frequent vascular invasion. This finding may implicate that tumor size was closely related to other prognostic factors in PCCA. For the other available prognostic factors by cox regression test involved in the nomogram, tumor grade was demonstrated as an independent prognostic factor, and showed strong impact on CSS of resected PCCA. Meanwhile, given main modification of the number of positive regional lymph nodes in the 8th edition guideline compared to 7 th TNM staging system, we set 3 as the cutoff value in positive regional lymph nodes to classify its impact on prognosis. On consistent with the 8th edition guideline, the number of positive regional lymph nodes severed as an independent factor in PCCA. Of note, we for the first time developed the nomogram for assess of the 1-, 3- and 5- year cancer-specific survival based on the tumor size. And our novel nomogram was validated to show the better accuracy than TNM staging system and might to help clinicians to develop personalized treatment strategies to improve the efficiency of medical resource allocation.

It is crucial to determine the generalizability and preventing overfitting of the novel prediction model by internal and external validation. The calibration plots for this study demonstrated the relatively good accurate between the nomogram predicted and actual observed 1-, 3- and 5-year CSS in both the SEER cohort and the external cohort. And compared with TNM stages, our 


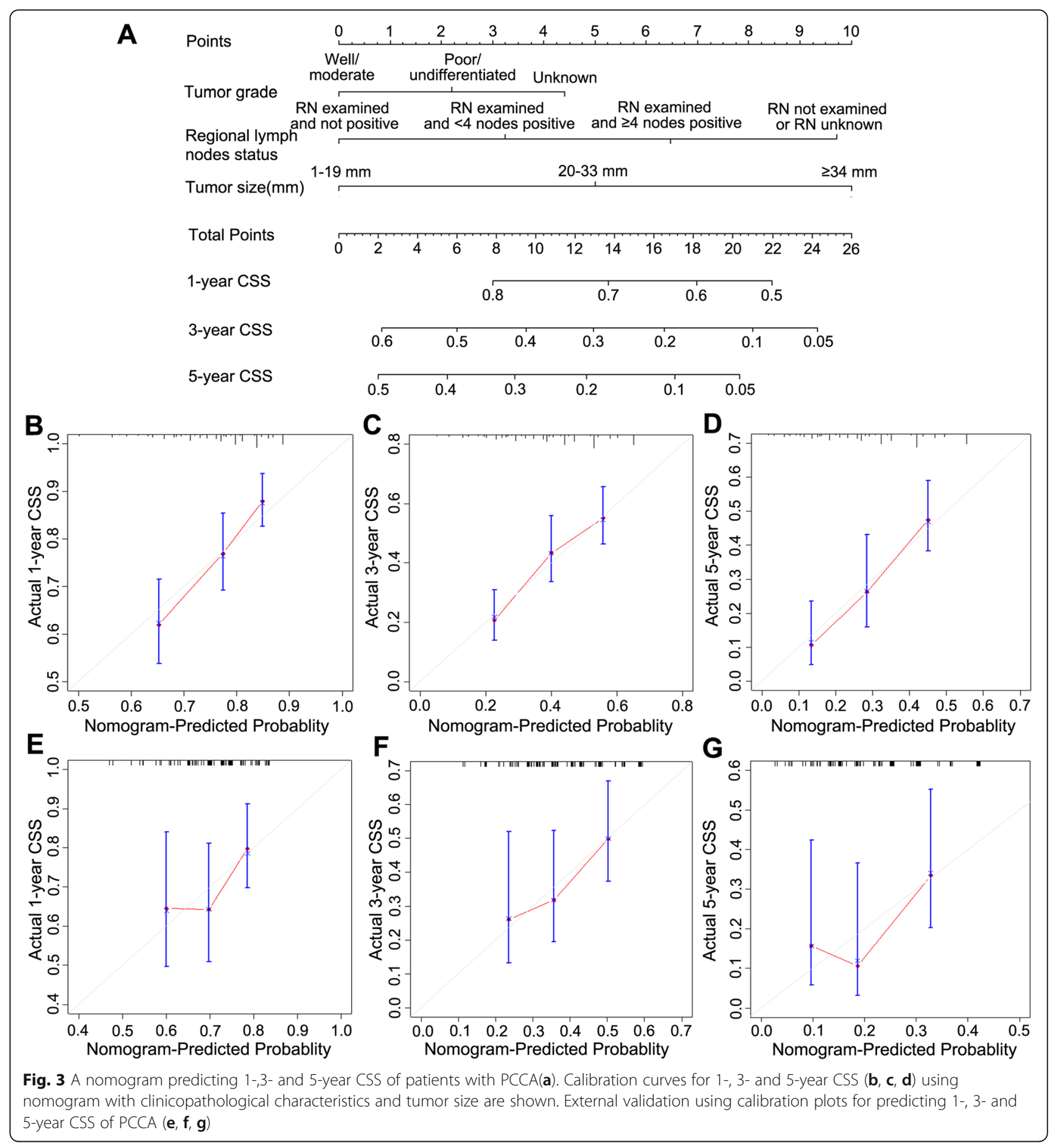

nomogram showed better predictive ability for prognosis of PCCA patients. Remarkably, our nomogram strengthened again the role of tumor size in influencing the survival of PCCA patients underwent resection.

Although this study was based on a large-scale population, there were still several potential limitations. First, SEER database did not record details in other cancer treatments (palliative therapy, radiation, chemotherapy), which play the important roles in the prognosis of resected PCCA. Second, some meaningful manifestations, such as resection margins, liver function and perineural invasion, are not involved in SEER. Third, considering the balance between comprehensibility and comprehensiveness, we only chose the clinical characteristics with high reproducibility and low time-varying. 


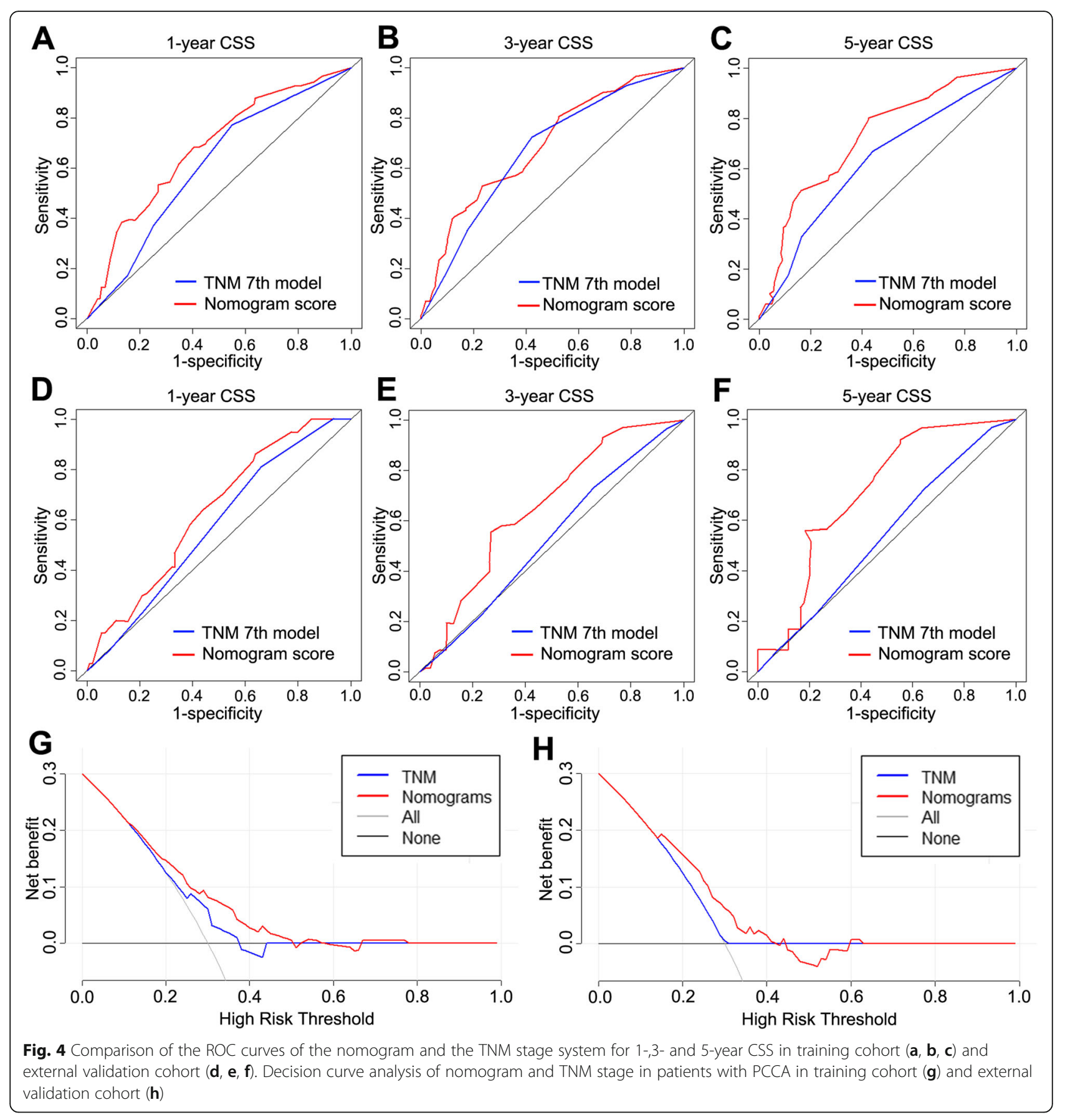

\section{Conclusions}

In conclusion, we demonstrated that tumor size was an independent factor in predicting the CSS of patients with PCCA. Increased tumor size was associated with worse tumor grade, advanced $\mathrm{T}$ stage, more positive regional lymph nodes and more frequent vascular invasion in PCCA. The proposed nomogram, which we constructed and external validated, might be useful to assist clinicians in predicting an individual's prognosis and planning treatment and follow-up schedules.

\section{Abbreviations}

HR: Hazard ratio; PCCA: Perihilar Cholangiocarcinoma; SEER: Surveillance, Epidemiology, and End Results; DCA: Decision Curve Analysis; AUC: The area under receiver operating characteristic curve; RN: Regional lymph nodes; ROC: Receiver operating characteristic; TNM: Tumor-Node-Metastasis; AJCC: The American Joint Commission on Cancer; CSS: Cancer-specific survival

\section{Acknowledgements}

We are very grateful to the staff in Surveillance, Epidemiology, and End Results Program (SEER) for their kind work in data collection and delivery. 


\section{Authors' contributions}

Conception or design were provided by XCL and YDZ. Acquisition, analysis, or interpretation of data were supplied by YDZ, ZSW and XW. Drafting of the manuscript was done by YDZ and CXL. Critical revision of the manuscript for important intellectual content was imparted by JC and WJJ. Statistical analysis was contributed by HWW. Administrative, technical, or material support was provided by YRW. All authors have given the final approval of the manuscript for submission and publication.

\section{Funding}

This study was supported by Natural Science Foundation of Jiangsu Province, China (BK20171077) and Key research and development program of Jiangsu Province (BE2016789), National Science Foundation of China (NSFC) (81700572, 81670570), National Science and Technology Major Project of China (2017ZX10203207-004-004). The funder had no involvement in study design; in the collection, analysis, or interpretation of data; in the writing of the report; or in the decision to submit the paper for publication.

\section{Availability of data and materials}

The data that support the findings of this study are available from Surveillance, Epidemiology, and End Results (SEER) Program of the National Cancer Institute (https://seer.cancer.gov).

\section{Ethics approval and consent to participate}

The investigation was approved by the ethics committee of the First Affiliated Hospital of Nanjing Medical University. Consent was waived considering the anonymous, observational, population-based, and registrybased nature.

\section{Consent for publication}

Not applicable.

\section{Competing interests}

The authors declare no potential conflicts of interest.

\section{Author details}

'Key Laboratory on Living Donor Transplantation, Ministry of Health, Department of liver surgery, The First Affiliated Hospital of Nanjing Medical University, 300\# Guangzhou Road, Nanjing 210029, Jiangsu Province, China. 2Department of Hepatobiliary and Laparoscopic Surgery, Yixing People's Hospital, Yixing, Jiangsu Province, China.

Received: 30 March 2020 Accepted: 6 October 2020

Published online: 30 October 2020

\section{References}

1. Nakeeb A, Pitt HA, Sohn TA, et al. Cholangiocarcinoma. A spectrum of intrahepatic, perihilar, and distal tumors. Ann Surg. 1996;224(4):463-73 discussion 473-5.

2. Rizvi S, Gores GJ. Pathogenesis, diagnosis, and management of cholangiocarcinoma. Gastroenterology. 2013;145(6):1215-29.

3. Wang Y, Li J, Xia Y, et al. Prognostic nomogram for intrahepatic cholangiocarcinoma after partial hepatectomy. J Clin Oncol. 2013;31(9): 1188-95.

4. Ribero D, Pinna AD, Guglielmi A, et al. Surgical approach for long-term survival of patients with intrahepatic Cholangiocarcinoma: a multiinstitutional analysis of 434 patients. Arch Surg. 2012;147(12):1107-13.

5. de Jong MC, Nathan H, Sotiropoulos GC, et al. Intrahepatic cholangiocarcinoma: an international multi-institutional analysis of prognostic factors and lymph node assessment. J Clin Oncol. 2011;29(23): 3140-5.

6. Farges $\mathrm{O}$, Fuks $\mathrm{D}$, Boleslawski $\mathrm{E}$, et al. Influence of surgical margins on outcome in patients with intrahepatic cholangiocarcinoma: a multicenter study by the AFC-IHCC-2009 study group. Ann Surg. 2011;254(5):824-9 discussion 830.

7. Jiang W, Zeng ZC, Tang ZY, et al. A prognostic scoring system based on clinical features of intrahepatic cholangiocarcinoma: the Fudan score. Ann Oncol. 2011;22(7):1644-52

8. Nathan $\mathrm{H}$, Pawlik TM, Wolfgang $\mathrm{CL}$, et al. Trends in survival after surgery for cholangiocarcinoma: a 30-year population-based SEER database analysis. J Gastrointest Surg. 2007;11(11):1488-96 discussion 1496-7.
9. Jarnagin WR, Bowne W, Klimstra DS, et al. Papillary phenotype confers improved survival after resection of hilar cholangiocarcinoma. Ann Surg. 2005;241(5):703-12 discussion 712-4.

10. Hemming AW, Mekeel K, Khanna A, et al. Portal vein resection in management of hilar cholangiocarcinoma. J Am Coll Surg. 2011;212(4):60413 discussion 613-6.

11. Ito F, Agni R, Rettammel RJ, et al. Resection of hilar cholangiocarcinoma: concomitant liver resection decreases hepatic recurrence. Ann Surg. 2008; 248(2):273-9.

12. Jarnagin WR, Fong $Y$, DeMatteo RP, et al. Staging, resectability, and outcome in 225 patients with hilar cholangiocarcinoma. Ann Surg. 2001; 234(4):507-17 discussion 517-9.

13. Ramacciato G, Nigri G, Bellagamba $R$, et al. Univariate and multivariate analysis of prognostic factors in the surgical treatment of hilar cholangiocarcinoma. Am Surg. 2010;76(11):1260-8.

14. DeOliveira ML, Cunningham SC, Cameron JL, et al. Cholangiocarcinoma: thirty-one-year experience with 564 patients at a single institution. Ann Surg. 2007;245(5):755-62

15. Chaiteerakij R, Harmsen WS, Marrero CR, et al. A new clinically based staging system for perihilar cholangiocarcinoma. Am J Gastroenterol. 2014; 109(12):1881-90.

16. Cheng QB, Yi B, Wang JH, et al. Resection with total caudate lobectomy confers survival benefit in hilar cholangiocarcinoma of bismuth type III and IV. Eur J Surg Oncol. 2012;38(12):1197-203.

17. Regimbeau JM, Fuks $D$, Pessaux $P$, et al. Tumour size over $3 \mathrm{~cm}$ predicts poor short-term outcomes after major liver resection for hilar cholangiocarcinoma. By the HC-AFC-2009 group. HPB (Oxford). 2015;17(1):79-86.

18. Hakeem AR, Marangoni G, Chapman SJ, et al. Does the extent of lymphadenectomy, number of lymph nodes, positive lymph node ratio and neutrophil-lymphocyte ratio impact surgical outcome of perihilar cholangiocarcinoma? Eur J Gastroenterol Hepatol. 2014;26(9):1047-54.

19. Mao K, Liu J, Sun J, et al. Patterns and prognostic value of lymph node dissection for resected perihilar cholangiocarcinoma. J Gastroenterol Hepatol. 2016;31(2):417-26

20. Nishio H, Nagino M, Nimura Y. Surgical management of hilar cholangiocarcinoma: the Nagoya experience. HPB (Oxford). 2005;7(4):259-62.

21. Ethun CG, Lopez-Aguiar AG, Anderson DJ, et al. Transplantation versus resection for Hilar Cholangiocarcinoma: an argument for shifting treatment paradigms for Resectable disease. Ann Surg. 2018;267(5):797-805.

22. Deoliveira ML, Schulick RD, Nimura Y, et al. New staging system and a registry for perihilar cholangiocarcinoma. Hepatology. 2011;53(4):1363-71.

23. Balachandran VP, Gonen M, Smith JJ, et al. Nomograms in oncology: more than meets the eye. Lancet Oncol. 2015;16(4):e173-80.

24. lasonos A, Schrag D, Raj GV, et al. How to build and interpret a nomogram for cancer prognosis. J Clin Oncol. 2008;26(8):1364-70.

25. Shariat SF, Karakiewicz Pl, Suardi N, et al. Comparison of nomograms with other methods for predicting outcomes in prostate cancer: a critical analysis of the literature. Clin Cancer Res. 2008;14(14):4400-7.

\section{Publisher's Note}

Springer Nature remains neutral with regard to jurisdictional claims in published maps and institutional affiliations.

Ready to submit your research? Choose BMC and benefit from:

- fast, convenient online submission

- thorough peer review by experienced researchers in your field

- rapid publication on acceptance

- support for research data, including large and complex data types

- gold Open Access which fosters wider collaboration and increased citations

- maximum visibility for your research: over $100 \mathrm{M}$ website views per year

At $\mathrm{BMC}$, research is always in progress.

Learn more biomedcentral.com/submission 